\title{
Advances in intelligence and internet of things for human-centric computing
}

\author{
Yi $\operatorname{Pan}^{1}\left[\right.$ ] Jong Hyuk Park ${ }^{2}$
}

Published online: 21 May 2018

(c) Springer-Verlag GmbH Germany, part of Springer Nature 2018

\section{Introduction}

Human-centric computing (Human-Com) is capable of sensing, monitoring, measuring, predicting and reacting intelligence to the real world. Remarkable advances in intelligence within information processing, mobile communications, and fuzzy systems are creating a new paradigm. In particular, recent advances in intelligence and Internet of things (IoT) have been creating many challenges to computer science and engineering. These frameworks might help support the interaction between "things" and human. It also allows for applying more complexed structures like fuzzy, neural networks, and cloud computing applications. Therefore, many issues need to be addressed in order to provide innovative change to Human-Com, which requires more research on intelligence and IoT technologies. In-depth study of the human experience that encompasses a broad range of research topics is an important challenge for improving diverse human-centric services.

This special issue is soliciting advanced articles addressing research outcomes and challenges in human experience understanding for better service provision in intelligence and IoT. The original and research articles addressed in various aspects include theoretical studies, advanced algorithm, new technology, and experimental prototypes. This special issue solicited innovative ideas and solutions in advanced intelligence and IoT for Human-Com. The general scope of this issue covers for Human-Com such as fusion algorithms, genetic programming, neural networks, cognitive science and engineering, security and privacy, and autonomous computing and efficiency modeling in IoT. It also includes new

$凶$ Jong Hyuk Park

jhpark1@seoultech.ac.kr

Yi Pan

yipan@gsu.edu

1 Georgia State University, Atlanta, GA 30302-5060, USA

2 Seoul National University of Science and Technology, Seoul, South Korea approaches for the linguistic processing, image processing, social relationship analysis, biometrics, and network security, among others. As a result, with careful consideration, this special issue selects and publishes excellent research articles from a large number of contributed papers.

\section{Related works}

Hao et al. (2017) proposed a novel approach for m-triadic concept-based algorithm for discovering the social evolution in location-based social online communities. The previous researchers in online social networks (OSNs) focused on social graph mining and analysis, which is based on the topology of the network such as dividing large graphs into the worthwhile cluster. Meanwhile, it is able to acquire meaningful result by elucidating the social weighted-graph that are derived from actual user locations in OSNs (Brown et al. 2012). Therefore, this paper describes exploiting the social evolution pattern from location-focused online communities by considering the dynamic feature in LBSs. Their approach can reduce the time-consuming problem efficiently.

It is a complexed and difficult work that performs cooperative tasks without central control in the intelligent swarm robotics application. De Rango et al. (2017) proposed a design to explore an unknown area using a stigmergy approach based on ants' behavior with a novel swarm, which is based on the protocol to volunteer and coordinate robots for disarming the mines with cooperation. The new stigmergy and ant swarm method can minimize robot recruiting, screening, and detection time. The proposed ant-based task robot coordination exploration algorithm (ATRC-OE) is compared with IAS-SS (Calvo et al. 2012), and it proved the improvement according to experiment result.

The automatic thresholding in visual inspection techniques is a common technique for detecting errors. It is due to the simplicity considering efficient implementation. Truong and Kim (2017) proposed an automatic thresholding technique based on Otsu's method with improvement using an 
entropy weighting scheme. The proposed method provides the effective detection performance even with remarkably small defect regions compared to the surface region.

Kim et al. (2017) proposed an automatic grading algorithm using Lexico-semantic pattern (LSP), which is an effective approach to analyzing rich Korean language morphologically. The postposition method in this paper provides a helpful toolkit for discovering the differences in the Korean language. In addition, they also applied a meaning extension based synonym dictionary to improve recall performance. They combined the ontology and word2vec technology with an automatic scoring method for a more efficient system. The experiments proved more accurate and efficient compared with other methods, namely, LSP, LSP-word2vec, and LSP-ontology.

Park and Jang (2017) proposed a novel approach to fingerprint recognition in order to identify counterfeit fingerprints. The proposed fingerprint detection is based on combinations of the statistical moment method, which is derived from fingerprint features, including deviation, kurtosis, skewness, variance, hyperflatness, and hyperskewness. Those multidimensional features are associated with the feature level by the support vector machine. The proposed method provided about $99 \%$ accuracy classification based on the simulation results.

To solve the problems in traditional approaches in data mining, the researcher pays attention to maximal frequent pattern mining (Yun and Lee 2016; Yun et al. 2016). This approach uses a small number of symbolic patterns to analyze it efficiently under a given dataset, unlike traditional approach extracts huge patterns based on threshold configuration. Lee and Yun (2017) surveyed recently announced maximal frequent pattern mining methods, which is based on comparing competitive elements and conducted performance evaluations to determine which method has a specific merit. As experiment results, the features of maximum frequency pattern mining can apply to the research fields such as sequential pattern mining and graph pattern mining. The proposed method is able to reduce the pattern minings and improve performance regarding memory usage and CPU runtime.

Lee et al. (2017) describes the design and implementation of atmospheric environment forecast accuracy using SARIMA-SVM (seasonal auto regressive integrated moving average-support vector machine) (Findley et al. 2016). The proposed algorithm is associated with the time series and nonlinear analysis to resolve the condition of atmospheric environment and enhance the accuracy of air pollution forecast. The proposed seasonal nonlinear time series algorithm enables the improvement of the detection accuracy for forecasting the atmospheric pollution. The experimental result showed a significant enhancement compared to the existing models.
Yin et al. (2017) designed an intrusion detection system (IDS) associated with data stream clustering algorithm for processing high-speed data stream. This proposed algorithm is divided into following two steps, online and offline. The online step acquires a summary of the given data stream. The summarized description and global parameters are maintained at regular intervals based on a sliding window dynamically. This approach can reduce storage space effectively and remove the sensitivity of parameters. The offline step reprocesses above-summarized description with an improved clustering algorithm to acquire clustering results. Experimental results show that the proposed system had efficient performance compared with traditional clustering algorithms regarding time and spatial complexity.

Jeong et al. (2017) proposed a new approach for the development of high performance on radar signal classifier based on the probability moment and ApEn. The proposed method is based on a signal that is given similar value. The differences from that are the changed order of the signal. Thus, it can effectively categorize the ambiguous radar signal in the existing system. The experiment performed simulations of 100 different types of radar signals in various bands. The performance result showed $99 \%$ rate of classification of 100 types of radar signals.

Doaa et al. (2017) suggested a new approach of the flower pollination algorithm (FPA) for solving the multidimensional knapsack problems called (MFPA). To solve MFPA, they use the sigmoid function handling discrete search space as a discretization method. This paper tested two datasets for benchmarking such as small-scale and large-scale MKPs to examine the performance compared with related works. The experimental results showed that the proposed algorithm could utilize both scales and provide better performance than the existing algorithms for large-scale MKPs.

Yao et al. (2017) proposed a coordinated control model to improve the route selection of emergency vehicles. The signal in this model provides coordinated tracking with different levels of traffic priority for the emergency situation. Shortening the travel time for emergency vehicles is an important issue to find the optimized emergency path from a rescue party to an incident location (Lee et al. 2015). The proposed model uses bi-level programming to solve this problem. The upper stage is designed to optimize the dynamic offsets, and the lower stage is to optimize the Green-Wave traffic theory. The proposed model is tested in micro traffic simulation software (PTV VISSIM) in comparison with the case without coordination. The proposed control method showed the effective validity of the experiment results.

In order for a machine to better utilize the meaning of the text, it is necessary to classify the entities appearing in the text into fine-grained types (Viswanathan and Krishnamurthi 2012). The existing methodologies typically have acquired entity context information through a fixed window, which 
may lead to ambiguous because there is not enough external information to classify the entity. To solve the drawbacks of this method, Liu et al. (2017a) proposed a detailed entity type classification method for unstructured text based on global information and sliding window context. By combining this information with other functions, this paper based on a bidirectional, short-term memory (LSTM) network to perform classification operations.

Liu et al. (2017b) proposed a novel approach for relation extraction model using dynamic LSTM network. This paper utilized features for the training a model including entity feature, entity position feature and POS (Part of Speech) feature. Based on above features in the relation extraction model, it enables handling complex relationships and enhancing the performance. The simulation results showed that it is an effective solution in classifying multiple relations among multiple entities. The precision level showed $72.9 \%$ in cases of three features, and they achieved $9 \%$ improvements based on dynamic LSTM.

Acknowledgements This work was supported by the National Research Foundation of Korea (NRF) grant funded by the Korea Government (MSIP) (No. 2016R1A2B4011069).

\section{Compliance with ethical standards}

Conflict of interest The authors declare that they have no conflict of interest.

\section{References}

Brown C, Nicosia V, Scellato S et al (2012) The importance of being location friends: discovering location-focused online communities. In: Proceedings of the 2012 ACM workshop on online social networks, pp 31-36

Calvo R, de Oliviera JR, Romero RAF (2012) A bio-inspired coordination strategy for controlling of multiple robots in surveillance tasks. Int J Adv Softw 5(3-4):146-165

De Rango F, Palmieri N, Yang X-S, Marano S (2017) Swarm robotics in wireless distributed protocol design for coordinating robots involved in cooperative tasks. Soft Comput. https://doi.org/10. 1007/s00500-017-2819-9

Doaa E-S, Mohamed A-B, Ibrahim E-H, Sang AK (2017) A modified flower pollination algorithm for the multidimensional knapsack problem-human centric decision making. Soft Comput. https:// doi.org/10.1007/s00500-017-2744-y

Findley DF, Lytras DP, Maravall A (2016) Illuminating ARIMA model-based seasonal adjustment with three fundamental seasonal models. J Span Econ Assoc 7:11-52
Hao F, Park D-S, Sim D-S, Kim MJ, Jeong Y-S, Park J-H, Seo H-S (2017) An efficient approach to understanding social evolution of location-focused online communities in location-based services. Soft Comput. https://doi.org/10.1007/s00500-017-2627-2

Jeong CM, Jung YG, Lee SJ (2017) Neural network based radar signal classification system using probability moment and ApEn. Soft Comput. https://doi.org/10.1007/s00500-017-2711-7

Kim J-E, Park K-N, Chae J-M, Jung S-Y (2017) Automatic grading system for short descriptive answer written in Korean using Lexico-semantic pattern. Soft Comput. https://doi.org/10.1007/ s00500-017-2772-7

Lee G, Yun U (2017) Performance and characteristic analysis of maximal frequent pattern mining methods using additional factors. Soft Comput. https://doi.org/10.1007/s00500-017-2820-3

Lee JK, Jeong YS, Park JH (2015) s-ITSF: a service based intelligent transportation system framework for smart accident management. Hum-centric Comput Inf Sci 5(1):34

Lee N-U, Shim J-S, Yong-WJ, Park S-C (2017) Design and implementation of the SARIMA-SVM time series analysis algorithm for the improvement of atmospheric environment forecast accuracy. Soft Comput. https://doi.org/10.1007/s00500-017-2825-y

Liu J, Wang L, Zhou M, Wang J, Lee S (2017a) Fine-grained entity type classification with adaptive context. Soft Comput. https://doi.org/ 10.1007/s00500-017-2963-2

Liu J, Ren H, Menglong W, Wang J, Kim H (2017b) Multiple relations extraction among multiple entities in unstructured text. Soft Comput. https://doi.org/10.1007/s00500-017-2852-8

Park Y, Jang UECL (2017) Statistical anti-spoofing method for fingerprint recognition. Soft Comput. https://doi.org/10.1007/s00500017-2707-3

Truong MTN, Kim S (2017) Automatic image thresholding using Otsu's method and entropy weighting scheme for surface defect detection. Soft Comput. https://doi.org/10.1007/s00500-017-2819-9

Viswanathan V, Krishnamurthi I (2012) Finding relevant semantic association paths through user specific intermediate entities. Humcentric Comput Inf Sci 2(1):1-11

Yao J, Zhang K, Yang Y, Wang J (2017) Emergency vehicle route oriented signal coordinated control model with two level programming. Soft Comput. https://doi.org/10.1007/s00500-017-2826-X

Yin C, Xia L, Zhang S, Sun R, Wang J (2017) Improved clustering algorithm based on high-speed network data stream. Soft Comput. https://doi.org/10.1007/s00500-017-2708-2

Yun U, Lee G (2016) Incremental mining of weighted maximal frequent itemsets from dynamic databases. Expert Syst Appl 54:304-327

Yun U, Lee G, Lee K (2016) Efficient representative pattern mining based on weight and maximality conditions. Expert Syst (in press)

Publisher's Note Springer Nature remains neutral with regard to jurisdictional claims in published maps and institutional affiliations. 